\title{
Blockchain HIE Overview: A Framework for Healthcare Interoperability
}

Brennan Bennett

Editor's note: This month, Telehealth and Medicine Today introduces a new department titled, Blockchain Technology Review, an ongoing series of articles by Mr. Brennan Bennett, which will report on emerging technologies in telehealth communications and health informatics applications. These initiatives offer tremendous opportunity to improve health outcomes by contributing innovative quantitative measures to the application of healthcare economics. Our goal is to increase understanding of the potential of blockchain among subscribers to Telehealth and Medicine Today.

Data stored in a blockchain is immutable and available for access by separate parties. The excellent potential residing in this technology includes security, verification, and expanded data management for healthcare records, making it ideal for a new interoperability standard.

\section{A Distributed Ledger for a Decentralized P2P Architecture}

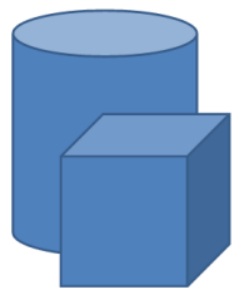

Centralized one node does everything

Image source: delphitools.info

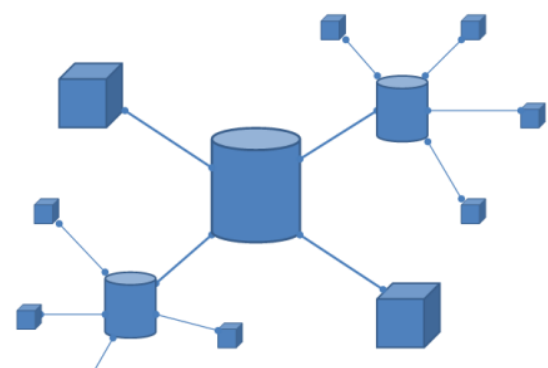

Distributed nodes distribute work to sub-nodes

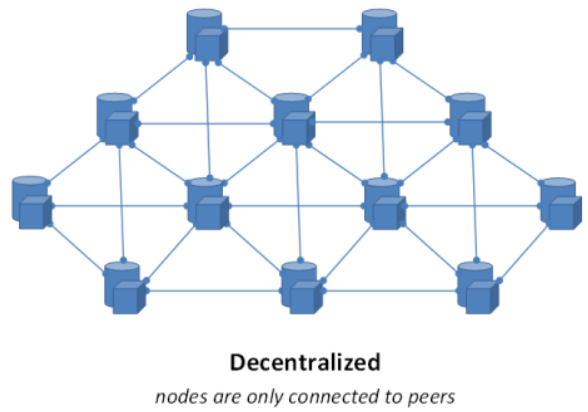

As it stands today, public blockchain technology (i.e. Bitcoin) is a secure P2P (peer-topeer) ledger system that uses public key encryption to protect information. Once entries are created on the chain, they are immutable, making blockchain ideal for storing permanent records. Because of this, authorized members of a network are confident of their data's authenticity within the encrypted chains. The shared ledger structure provides an immutable audit trail for every transaction. In healthcare, organizations can create authenticated records and entries without needing a central authority. Each link 
in the chain verifies the next, traceable back to what's called the Genesis block, a.k.a. the first block in the chain ever created.

While the origins of blockchain lie in P2P financial transactions, the technology shows excellent potential in healthcare applications that require trust to work efficiently, with information privacy is as the guiding principle. Ease of access, i.e. implementation simplification, is one of the biggest obstacles in the way of moving the needle from potential benefits to transformative technology. Before implementation occurs however, there must be a strategy that business leaders as well as developers can understand. Scott Hoch of the blockchain innovation company Gem, concludes that there are three key points to blockchain strategy development:

Our experience has shown that the three most important things to understand at an executive level are:

- What problems are blockchain uniquely suited to solve?

- What symptoms can identify these types of problems?

- What are non-cryptocurrency applications of blockchain that have been implemented today?

\section{Setting Healthcare Interoperability Standards with Blockchain}

A shared HIE (health information exchange) blockchain format removes the constraints that divide independent healthcare information systems and facilitate interoperability. A healthcare data management system that eliminates the boundaries between existing file systems makes electronic health records universally sharable while protecting the confidential information of patients.

Digital Inclusion through Trust and Agency is a blockchain standards program recently launched by The Institute of Electrical and Electronics Engineers, or IEEE. The core focus of the program is to fine tune standards around identity management to protect data privacy and data ownership.

Standards implementation in the Blockchain space will unleash new capabilities for trend analysis and ultimately action oriented preventative by not just processing data, 
but understanding the data its crunching. Currently, healthcare records are proprietary to the organizations that create them, due to a lack of national standards or shared information technology architecture. Access to non-identifying data will transform the way the healthcare industry delivers, records, and measures patient data.

\section{Tracking for Clinical Information in the Transaction Layer}

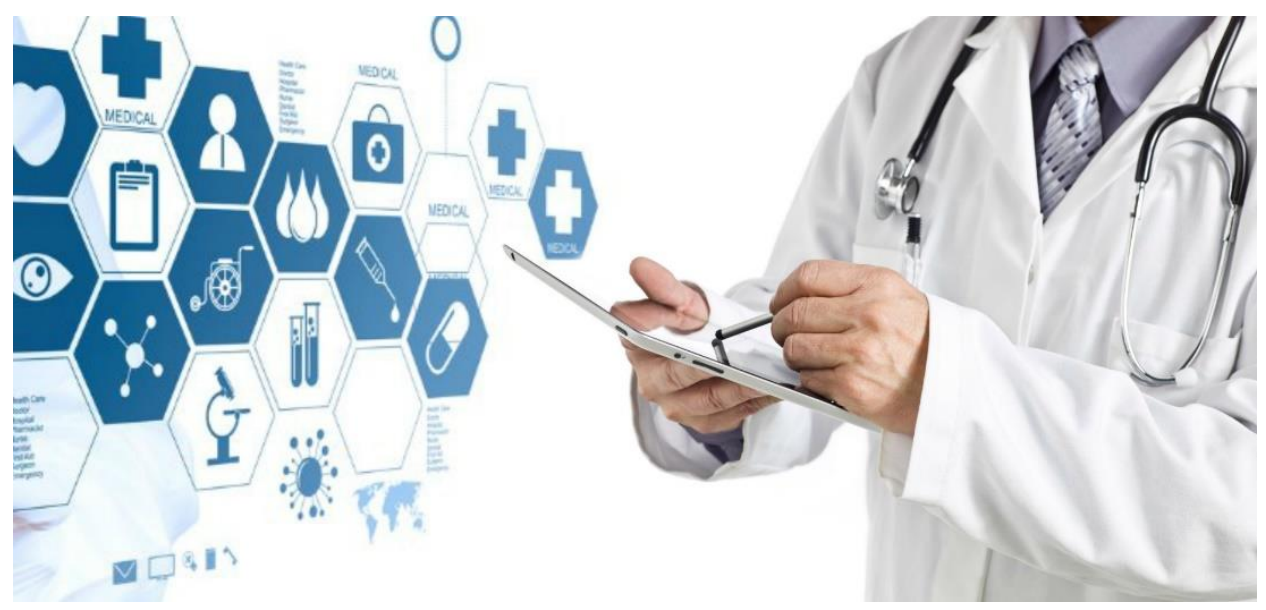

The transformative blockchain feature for HIE lies in the ability to attach a standard layer of encrypted information to each record. This transaction layer, for example, is a separate layer of information from the actual patient data. Transactions in this regard are not covered by privacy rules, but to protect some of the data, a blockchain HIE enabled system must protect all the data. Non-identifiable demographics and service information can be mined for research contribution, thereby incentivizing the patient to allow a certain level of access; but the patient must be the decider unto whether to share scrubbed clinical information.

A framework that includes this transaction layer provides globally available information, statistics, and useful demographics. Blockchain encryption stores two types of information in the transaction layer. It includes the first on-chain and then other off-chain encrypted links to sources on separate databases.

On-chain information is immediately available to users with the proper permissions, but the format limits its scope; the processing time slows as the size of the data increases. 
The off-chain information sources are limitless. Given the key, authorized recipients can access files of any format such as x-rays, or MRI (magnetic resonance imaging) scans. The small link keeps processing times to a minimum.

\section{Blockchain Makes It Harder to Hack Patient Information}

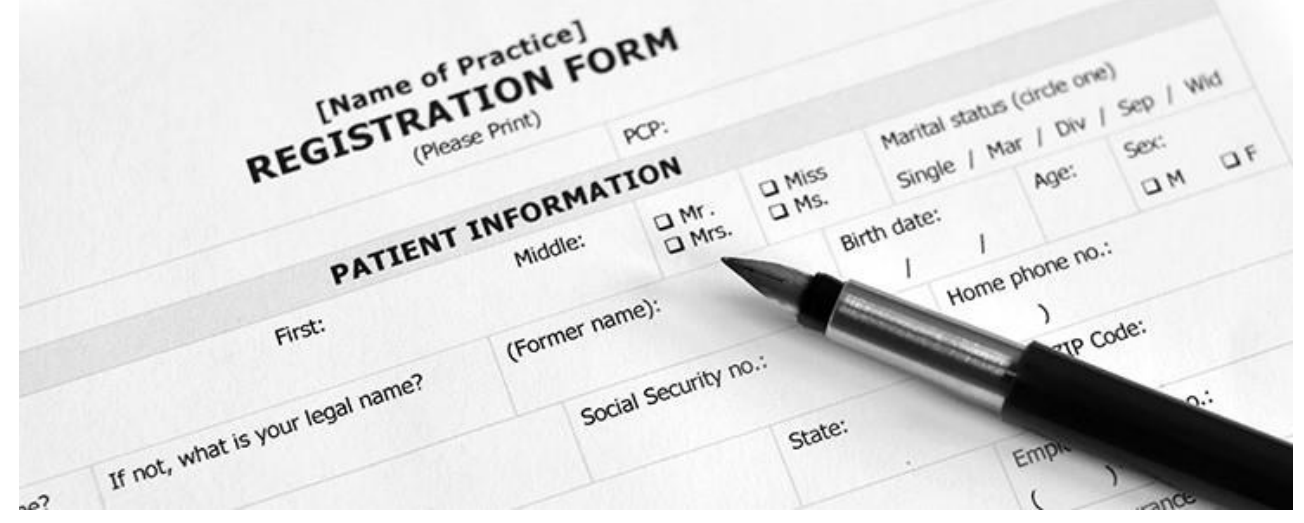

A standardized format for healthcare information will allow a smart contract, as well as define the approvals required before encoding in the blockchain. An interoperable blockchain standard strengthens integrity while sharing the data more widely. At the same time, the private information and patient identities are only available to authorized users.

Hacking the private key of a file limits the risk of damage and the extent of compromise to that single record. To compromise the entire blockchain system requires cracking the key for each file. This added layer of security is vital in the present age where cybercrime and ransomware are widespread. 


\section{APIs Match Unique Data to Standard Formats}

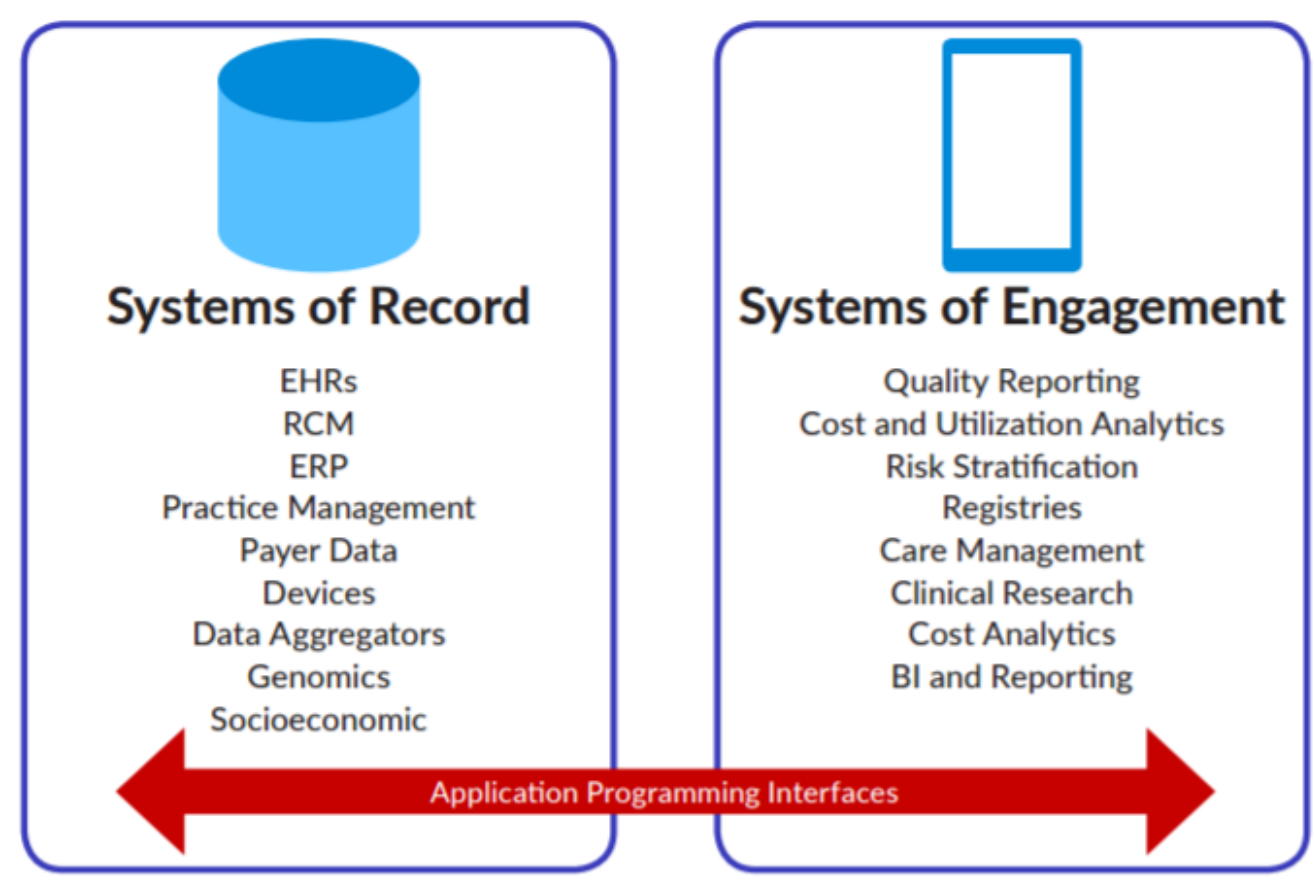

APIs Address Need for Better Systems of Record and Engagement

Image source: technative.io

All organizations, with permission, can have access to the information stored in blockchain through an API (application program interface) that acts as a gateway for standardized information. The use of APIs gives frictionless access to patient information that delivers patient interactions to the smart contract.

The ability to access non-identifiable patient information stored in the transaction layer makes for precision medicine. Blockchain integrates the relevant information without changing the underlying information that gives diverse users the ability to apply their most useful structures.

Blockchain HIE solutions can deliver optimal outcomes for healthcare organizations, but ultimately will be limited in scope until a full-scale industry adoption takes place. 


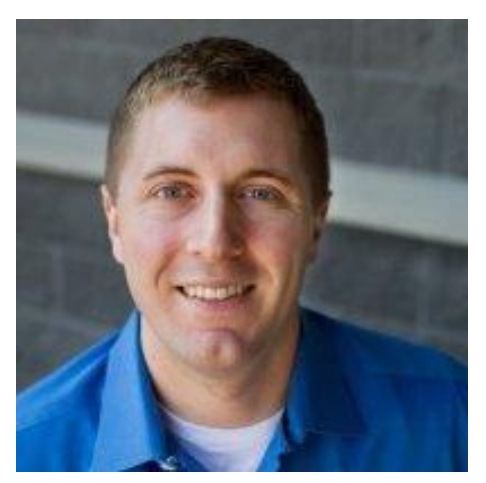

Brennan Bennett is the Founder and Managing Editor of Blockchain Healthcare Review, a publication covering technical innovations in blockchain technology and how it is reshaping the future of health data governance. He is an experienced HIT strategy consultant with a background in the Fortune 100 space. Additionally, Brennan holds a M.S. in Biomedical Informatics from Rutgers-The State University of New Jersey.

Department: Blockchain

Tags: Blockchain, health information exchanges, HIE, patient records, security 